\title{
Effect of the Tokamak Size in Edge Transport Modelling and Implications for DEMO
}

\author{
H. D. Pacher, ${ }^{\mathrm{a}, *}$, A. S. Kukushkin ${ }^{\mathrm{b}}$, G. W. Pacher ${ }^{\mathrm{c}}$, G. Janeschitz $^{\mathrm{d}}$, D. Coster ${ }^{\mathrm{e}}$, V. Kotov ${ }^{\mathrm{f}}$, D. \\ Reiter $^{\mathrm{f}}$ \\ ${ }^{a} I N R S-E M T$, Varennes, Québec, Canada \\ ${ }^{b}$ ITER International Team, Garching Joint Work Site, Garching, Germany \\ ${ }^{c}$ Hydro-Québec, Varennes, Québec, Canada \\ ${ }^{d}$ Forschungszentrum Karlsruhe, Karlsruhe, Germany \\ ${ }^{e}$ Max-Planck IPP, Garching, Germany
}

${ }^{f}$ FZ Jülich, Jülich, Germany

\begin{abstract}
The edge plasma of four devices, AUG, JET, ITER and a prototypical DEMO near ignition, is modelled with B2-EIRENE with a linear neutral model, carbon impurity, and variable throughput and scrape-off layer power. The scaling from ITER to DEMO is quantified as a power law scaling in device size, and JET and AUG simulations are compared to this scaling. Compared to ITER at the same scrape-off layer power per unit device volume and the same operating point relative to the edge-based density limit, edge helium density and peak power per unit area is found to be similar, and helium influx lower, for DEMO with impurity seeding. With a full neutral model, helium parameters are improved by a factor 3. Results of core modelling show that ignited operation of DEMO is possible with the impurity seeding required to render peak power loads compatible with a helium-cooled divertor.
\end{abstract}

JNM keywords: T0100, P0500, P0600 
PSI17 keywords: B2/EIRENE, Edge modelling, Edge pedestal, Fusion reactor, ITER -

PACS: 52.55.Fa, 28.52.-s, 52.65.-y, 52.55.Rk, 52.25.Vy

* Horst D. PACHER, INRS-EMT, 1650 boul. Lionel Boulet, Varennes QC J3X 1S2, Canada.

E-mail: pacher@emt.inrs.ca, tel. +1-450-929-8241, fax +1-450-929-8102 


\section{Introduction}

This paper investigates the extrapolation of divertor and core operation from ITER to DEMO, with particular emphasis on the scaling of the edge plasma parameters and the core impurity seeding required to attain acceptable peak power load on the divertor plates and acceptable helium density at ignited operation in DEMO. The geometric parameters chosen here for DEMO are similar to those of the ITER Conceptual Design Activity (1991), but with stronger triangularity $(B=5.7 \mathrm{~T}, \mathrm{R}=8.1 \mathrm{~m}, \mathrm{a}=2.8 \mathrm{~m}, \kappa=1.7, \delta=0.36)$. Further devices modelled are ASDEX Upgrade (AUG) Divertor II and IIb, and JET MkII. The size of the device is characterised by $\mathrm{R}_{\text {outer }}$, the major radius of the outer strike point. For AUGIIb, AUGII, JETMkII, ITER, and DEMO, $\mathrm{R}_{\text {outer }}$ is $1.61,1.71,2.90,5.55$, and $8.12 \mathrm{~m}$, respectively. In the $2 \mathrm{D}$ edge modelling, the size scaling of the results will be established from ITER to DEMO; the results from the other devices will be shown for comparison. The smaller devices deviate progressively from the scaling for high-power, large devices, probably because their ratio of neutral mean free path to machine size is not negligible and they therefore have larger neutral densities at, and much larger neutral fluxes across, the separatrix.

\section{Edge and divertor modelling of ITER and DEMO, including also AUG and JET}

The edge and divertor plasma is modelled with the B2-Eirene code package, version solps4.2, employing a full multi-fluid description of the electrons and $\mathrm{D}, \mathrm{He}$, and $\mathrm{C}$ ions $[1,2]$. For most of the studies here, computational time limitations restricted our study to the linear neutral package (neither neutral-neutral collisions nor molecular kinetics) in toroidal geometry in EIRENE, but several studies were performed with the more complete non-linear Monte-Carlo neutral model as in [3]. The impurity other than helium in the edge simulations is carbon, selfconsistently generated at the divertor targets and the walls; impurity seeding in the core plasma is only included by varying the power $\mathrm{P}_{\mathrm{SOL}}$ into the scrape-off layer.

The results of the simulations are expressed, following [1-3] which established this as the key parameter for characterising the edge plasma operational point, as a function of the neutral pressure normalized to 1 at detachment of the inner divertor and given by 


$$
\mu \equiv \mathrm{p}_{\mathrm{DT} \#} \mathrm{P}_{\#}^{-0.87} \mathrm{f}_{\mathrm{f}}^{-0.8} \mathrm{f}_{\mathrm{w}}^{-1} \mathrm{q}_{95 \#}^{-0.27} \mathrm{f}_{\mathrm{nn}}^{-1} \mathrm{R}_{\#}^{-1.21} \mathrm{f}_{\mathrm{He}}^{0}
$$

Here $\mathrm{p}_{\mathrm{DT} \#}$ is the average divertor neutral pressure at the entrance to the private flux region, normalised (indicated by \#) to its value at the incipient detachment. $\mu$ is normalised by powers of $\mathrm{P}_{\mathrm{SOL}}$ and the safety factor, as well as corrections by factors describing the type of fuelling (usually a small correction for gas puffed simulations), the type of wall (carbon or realistic [9]) and the neutral model (linear, neutral-neutral collisions, full model). The fuelling factor, $\mathrm{f}_{\mathrm{f}} \equiv 1+0.18 \Gamma_{\text {core }} / \Gamma_{\mathrm{DT}}$, relates the flux fuelling the core directly to the total throughput. The wall factor $\mathrm{f}_{\mathrm{w}}$ is 0.84 for the full carbon walls [9]. The factor $\mathrm{f}_{\mathrm{nn}}$ related to the neutral model is 1 for the linear neutral model and 1.83 for the full neutral model which yields higher $\mathrm{p}_{\mathrm{DT} \#}$ at detachment [3]. The normalisations and exponents of the scalings are given in Table I; the column giving the size scaling is the object of this section. The devices are compared at equivalent SOL power per unit device volume and specific pumping speed, i.e. these quantities are now defined as:

$$
\mathrm{P}_{\#} \equiv \mathrm{P}_{\mathrm{SOL}} / 100 \mathrm{R}_{\#}^{3}, \mathrm{~S}_{\#} \equiv \mathrm{S} / 114 \mathrm{R}_{\#}^{2}, \text { where } \mathrm{R}_{\#} \equiv \mathrm{R}_{\text {outer }} / \mathrm{R}_{\text {outer_ITER }}
$$

and, in contrast to [1], the pumping speed is now the engineering pumping speed at the duct entrance (for further discussion see [8]), which has a maximum value of $75 \mathrm{~m}^{3} / \mathrm{s}$ in ITER. The devices are compared at a ratio of helium production rate to $\mathrm{P}_{\mathrm{SOL}}$ corresponding to $\mathrm{Q}=10$ and core radiation fraction of $30 \%$; the factor $\mathrm{f}_{\mathrm{He}}$ applied to helium densities and fluxes is then 1 . For different $\mathrm{Q}$ and radiation fraction, $\mathrm{f}_{\mathrm{He}}$ becomes $\mathrm{f}_{\mathrm{He}}=1.05 \mathrm{P}_{\alpha} / \mathrm{P}_{\mathrm{SOL}}[2]$.

Incipient detachment of the inner divertor is signalled by a sudden drop of the maximum electron temperature at the inner divertor as $\mathrm{p}_{\mathrm{DT}}$ is raised, and either a flattening of the curve of DT density at the separatrix and a steepening of the curve of DT neutral flux across the separatrix or both; the detachment point is taken to be the lowest pressure of these. The corresponding normalized pressure is shown as a function of device size in Fig.1 and is seen to scale well with device size for JET, ITER and DEMO, as $\mathrm{R}_{\#}^{1.21}$, the value given by anticipation in Eq. (1). The operating window over which the scalings hold typically extends from $\mu=1$ to $\mu \approx 0.5$; its scaling with input parameters can not be discussed here. 
Fig. 1 also shows the scaling of the average DT density at the separatrix at the incipient detachment point $\mu=1$ versus machine size, and Fig. 2 the DT neutral influx to the core across the separatrix. Both increase linearly as $\sim R_{\#}^{1}$ with device size at equivalent power per unit volume and specific pumping speed (Eq. (2)). The smaller devices deviate significantly from this: the AUG density is much higher than the scaling and the JET density, while closer to the scaling, still exceeds it, probably because the ratio of neutral mean free path to machine size is significant in the smaller devices but not in ITER and DEMO. Geometry can influence the scaling, as shown for example by the study contrasting domelss configuration and configurations with various dome lengths in [8]. The present study is restricted to typical or reference geometries of the four devices, in particular for oblique angles of the divertor target to the magnetic surfaces in the case of ITER and DEMO.

At pressures below detachment (not shown), these two parameters follow the same scaling with parameters other than size as ITER. This is generally true for the parameters discussed in this section.

Fig. 2 shows the scaling of neutral He influx to the core across the separatrix and Fig. 3 the scaling of average He density at the separatrix at incipient detachment $\mu=1$. In contrast to [1], both now have moderate power dependence, given in Table I. It is seen that the inward neutral helium flux across the separatrix decreases strongly (as $\mathrm{R}_{\#}^{-1.46}$ ) and therefore the helium influx per unit area decreases very strongly. The scaled helium density (Fig. 3) barely increases with size (as $\mathrm{R}_{\#}^{0.15}$ ). Both of these conditions enter into the boundary conditions for the core modelling, and DEMO is actually improved over ITER. These results apply to the linear neutral model, and are further improved with the full neutral model (see below).

Fig. 3 also shows the positive result that the normalised peak power load per unit area (at the outer divertor, where it is higher), including radiation, at the detachment point remains practically constant from ITER to DEMO. The range covered in $\mathrm{MW} / \mathrm{m}^{2}$ for the range of ITER powers is the same as that for the range of DEMO powers, which are higher. 
The carbon impurity density at the separatrix and total erosion flux at the target are found to scale as $R_{\#}^{-0.6}$ and $R_{\#}^{2.5}$, respectively. Since the area subject to erosion increases as $R_{\#}^{1}$ times the scrape-off width at the target, and we find the latter to increase as $R_{\#}^{1.6}$, the average erosion depth per unit time, and thus lifetime of a carbon divertor, remains approximately constant from ITER to DEMO. DEMO would require a larger lifetime, but is in any case likely to use a material other than carbon, to be determined in future. The radiated power increases as $\mathrm{R}_{\#}^{3.3}$, and the fraction of $\mathrm{P}_{\mathrm{SOL}}$ deposited at the target drops from $70 \%$ in ITER to 50\% in DEMO. The electron and ion temperatures at the separatrix vary as $\mathrm{R}_{\#}^{-0.64}$.

At the present time, only a limited number of simulations with the complete neutral model $[3,8]$ exist for ITER, and even fewer for DEMO which are in some cases less well converged for now. However, to determine the actual pumping for DT or He the full neutral model must be used because the linear neutral model, lacking neutral-neutral collisions, can not properly represent the density and temperature distributions of the neutrals in the volume between the edge of the plasma grid and the pump duct entrance. Previous studies for ITER have shown [3] that $\mathrm{p}_{\mathrm{DT} \#}$ at detachment is higher $\left(f_{n n}\right.$ in Eq. (1)), but most of the quantities, with the exception of the carbon erosion and the helium quantities, have a similar value and scaling at the same detachment state for the linear and the complete neutral models, e.g. the peak power per unit area. With the nonlinear neutral transport model at the same engineering pumping speed at the pump duct, the ratio of helium pressure to throughput at the grid edge turns out to be lower for He than for DT (i.e. the "pumping speed" at the grid edge is higher for helium than for DT) and therefore the helium pumping from the divertor is more efficient than could be deduced from the linear model. This is shown in Fig. 4: for both ITER and DEMO, the helium density and neutral influx are a factor 3 lower than from the linear neutral model. This additional factor is shown in Table I; its scaling, if any, remains to be determined. The DT influx increases by a factor 2 but remains low for ITER and DEMO. 


\section{Modelling of the core plasma of DEMO}

Core plasma operation of DEMO was simulated using the 1.5 D Astra transport code. The theory-based transport model is similar to that previously used for ITER studies [4], with MMM energy transport [5], a pedestal created by ExB and magnetic stabilisation, anomalous particle transport, and time-averaged ELMs (see [4]) and calibrated to JET and AUG experimental results. Neoclassical impurity transport [6] is added to turbulence-driven transport equal for all species, impurities include helium, carbon and a seeded impurity (carbon, unlikely to be used in DEMO, is maintained to retain some low-Z impurity). Several impurity transport models and seed impurities are being modelled [7] - here space permits only the presentation of neoclassical transport and Xenon impurity.

The separatrix DT, He, and C densities, separatrix ion and electron temperatures, and separatrix inward neutral DT and He fluxes are taken from the scaling relations above with the core simulation quantities as input. The neutral DT temperature is set to one-half the separatrix ion temperature and the neutral helium temperature is set to $30 \mathrm{eV}$. The control parameters for the core simulation are then the core fuelling flux $\Gamma_{\text {core }}$ ("pellet" fuelling, adjusted to give the desired core density), the gas puff flux into the vessel $\Gamma_{\text {puff, }}$, the additional heating power $P_{\text {aux }}$ (kept small to have near-ignited operation at $\mathrm{Q}=150$ ), and the edge density of the seed impurity $n_{z, \text { edge }}$. The fusion power was kept constant, and the operating point was kept at $\mu=0.75$.

Fig. 5 shows $\mathrm{P}_{\mathrm{SOL}}$ and the core density as a function of seed impurity concentration. The impurity seeding reduces $\mathrm{P}_{\mathrm{SOL}}$ to alleviate divertor loading and the core density rises slightly, remaining near the Greenwald limit but $25 \%$ below the edge-based limit $\mu=1$, The dilution is low, $\mathrm{Z}_{\text {eff }}$ remains below 2.5, and the core helium concentration is in the range 6-8\% (not yet using the factor $1 / 3$ discussed at the end of the last section). Fig. 6 shows that the normalised peak power load remains the same for all devices as a function of $\mu$. The same figure shows that impurity seeding has reduced $\mathrm{P}_{\mathrm{SOL}}$ sufficiently to attain a peak power of $7 \mathrm{MW} / \mathrm{m}^{2}$ in nearlyignited DEMO operation. 


\section{Conclusions}

An integrated core and edge/divertor model, linked [4] at the separatrix via scaling relations derived from a large number of edge/divertor runs, has been applied to a plasma prototypical of DEMO ELM'y H-mode at conditions close to ignition with medium- to high-Z impurity seeding employed to reduce the power into the scrape-off layer and thereby the divertor power load.

The edge/divertor modelling with B2-EIRENE and the linear neutral model shows DEMO parameter scaling similar to ITER's. It is found that at the same SOL power per unit volume of the device, and the same specific pumping speed for ITER and DEMO, the separatrix helium density remains constant, the helium neutral influx decreases, and the peak power loading of the divertor plates remains constant in the transition from ITER to DEMO - all positive results.

The sparse results available at present with the full neutral model indicate that the peak power does not change, but the helium density and influx are factor 3 lower than from the linear neutral model - a very positive result for both ITER and DEMO. The neutral DT influx increases by a factor 2 but remains low for both.

The core simulations using the edge size scaling were carried out with a model previously calibrated to AUG and JET. Impurity seeding was employed to reduce the power into the SOL and thereby the divertor power load while the fusion power was held at the desired value by adjusting the fuelling rate and thereby raising the plasma density as the impurity concentration increased. Close-to-ignited operation $(\mathrm{Q}=150)$ of $\mathrm{DEMO}$ was found to be feasible at a fusion power of $3 \mathrm{GW}$, at $75 \%$ of the edge density limit based on divertor detachment, and with a peak divertor power load of $7 \mathrm{MW} / \mathrm{m}^{2}$. The power entering the SOL ( 300 MW) was still well above the LH threshold. Such impurity seeded near-ignition operation is feasible over a reasonable operating window and is consistent with use of a helium-cooled divertor, which requires a peak power below $10 \mathrm{MW} / \mathrm{m}^{2}$.

Further simulations are needed to clarify the existing caveats. SOL scaling with seeded impurity (rather than C), full neutral model, and mixed-material surfaces needs to be established over a wider parameter range. Core modelling needs to include validation and variation of impurity 
transport models, longer pulses and hybrid scenarios, and to incorporate the future seed impurity scaling from the SOL model.

\section{References}

[1] Pacher H.D., Kukushkin A.S., Pacher G.W., et al J. Nucl. Mat. 313-316C (2003) 657

[2] Kukushkin A.S., Pacher H.D., Pacher G.W., Janeschitz et al., Nucl. Fusion 43 (2003) 716

[3] Kukushkin, A. S., Pacher, H. D., Kotov, V., Reiter, D., et al Nucl. Fusion 45 (2005) 608

[4] Pacher G.W., Pacher H.D., Janeschitz G., et al, Plasma Phys. and Contr. Fus. 46 (2004) A 257

[5] G. Bateman and A. H. Kritz, http://w3.pppl.gov/NTCC/MMM95/

[6] Houlberg, W., http://w3.pppl.gov/rib/repositories/NTCC/objects/Asset/nclass.html

[7] Janeschitz, G., Pacher, G.W., Pacher, H.D., Kukushkin, A.S., Proc. $33^{\text {rd }}$ EPS Conf. on Plasma Physics,, (2006), paper P1.110

[8] Kukushkin, A. S., Pacher, H. D., Kotov, V., et al, 17th Int.Conf. on Plasma Surface Interactions in Controlled Fusion Devices, PSI Hefei, (2006), subm. to J. Nucl. Mater.

[9] A.S. Kukushkin, H.D. Pacher, D. Coster, et al., J. Nucl. Mater. 337-339 (2005) 50 
Tables

\begin{tabular}{|c|c|c|c|c|c|c|c|c|c|c|c|c|}
\hline & & & $\mu$ & $\mathrm{f}_{\mathrm{He}}$ & $f_{f}$ & $\begin{array}{l}S_{\text {eng }} \\
{[S]}\end{array}$ & $\begin{array}{c}P_{S O L} \\
{[\mathrm{P}]}\end{array}$ & $\xi_{\mathrm{ei}}$ & $f_{w}$ & $q_{95}$ & $\mathrm{f}_{\mathrm{nn}}$ & $\mathrm{R}_{\#}$ \\
\hline & scale & $\begin{array}{r}\text { Full } \\
\text { neut. } \\
\text { model } \\
\text { factor }\end{array}$ & 8.5 & 1 & 1 & $\begin{array}{r}114 \\
\mathrm{R}_{\#}^{2}\end{array}$ & $\begin{array}{r}100 \\
\mathrm{R}_{\#}^{3}\end{array}$ & 1 & 1 & 3 & & 5.55 \\
\hline & & & \multicolumn{10}{|c|}{ exponents } \\
\hline $\mathrm{q}_{\mathrm{pk}}[\mathrm{q}]$ & 3.8 & 1 & -1.17 & 0 & -0.6 & 0 & 1.26 & 0 & -0.37 & -0.5 & 0 & -0.02 \\
\hline$n_{D T_{-} \text {sep }}[\mathrm{n}]$ & 0.3 & 1 & 0.43 & 0 & 0.8 & 0 & 0.55 & 0.05 & 0.68 & -0.5 & 0 & 1.02 \\
\hline $\begin{array}{l}\Gamma_{D T \_n \_s e p} \\
{[\Gamma]}\end{array}$ & 15 & 2 & 0.36 & 0 & -4.3 & 0.3 & 0 & 0 & 0.44 & -0.2 & 0 & 1.03 \\
\hline$T_{e_{-} \text {sep }}[\mathrm{eV}]$ & 170 & 1 & -0.12 & 0 & -0.2 & -0.02 & 0.32 & 0.049 & -0.16 & 0.5 & 0 & 0.64 \\
\hline$T_{i_{-} \text {sep }}[\mathrm{eV}]$ & 300 & 1 & -0.24 & 0 & -0.6 & -0.04 & 0.36 & -0.115 & -0.3 & 0.5 & 0 & 0.64 \\
\hline $\begin{array}{l}n_{C_{-} \text {sep }}[\mathrm{n}] \\
\text { realistic }\end{array}$ & 0.0056 & 1 & 0 & 0 & 0 & 0 & 0.54 & -0.13 & -0.77 & -0.32 & 0 & -0.6 \\
\hline $\begin{array}{l}n_{C_{-} \text {sep }}[\mathrm{n}] \\
\text { full carbon }\end{array}$ & 0.0067 & 1 & 0 & 0 & 0 & 0 & 0.54 & -0.13 & -0.77 & -0.32 & 0 & -0.6 \\
\hline $\begin{array}{l}\Gamma_{C_{-} \text {targ }}[\Gamma] \\
\text { realistic } \\
\end{array}$ & 230 & 1 & 0.58 & 0 & 0 & 0 & 0.75 & 0 & 3.4 & -0.58 & 0 & 2.5 \\
\hline $\begin{array}{l}\Gamma_{C_{-} \text {targ }}[\Gamma] \\
\text { full carbon }\end{array}$ & 360 & 1 & 0.58 & 0 & 0 & 0 & 0.75 & 0 & 3.4 & -0.58 & 0 & 2.5 \\
\hline$P_{i m p \_r a d}[\mathrm{P}]$ & 55 & 1 & 0.14 & 0 & 0 & 0 & 1.35 & 0 & -0.29 & 0.28 & 0 & 3.28 \\
\hline $\begin{array}{l}n_{H e \_s e p}[\mathrm{n}], \\
\mu>0.85\end{array}$ & 0.0027 & 0.33 & -2 & 1 & -4 & -1 & 0.79 & -0.1 & 0 & -0.85 & 0 & 0.15 \\
\hline $0.6<\mu<0.85$ & 0.0038 & 0.33 & 0 & 1 & -4 & -1 & 0.79 & -0.1 & 0 & -0.85 & 0 & 0.15 \\
\hline$\mu<0.6$ & $\begin{array}{r}0.0013 \\
5 \\
\end{array}$ & 0.33 & -2 & 1 & -4 & -1 & 0.79 & -0.1 & 0 & -0.85 & 0 & 0.15 \\
\hline $\begin{array}{l}\Gamma_{H e \_n \_s e p} \\
{[\Gamma], \mu>0.85}\end{array}$ & 0.26 & 0.33 & -2 & 1 & -2 & -1 & 0.22 & 0 & 0 & 0 & 0 & -1.46 \\
\hline $0.6<\mu<0.85$ & 0.36 & 0.33 & 0 & 1 & -2 & -1 & 0.22 & 0 & 0 & 0 & 0 & -1.46 \\
\hline$\mu<0.6$ & 0.13 & 0.33 & -2 & 1 & -2 & -1 & 0.22 & 0 & 0 & 0 & 0 & -1.46 \\
\hline
\end{tabular}

Table I. Scaling of Divertor Parameters at Given Normalised Pressure (given detachment state)

for pressures lower than incipient detachment. Column 1: Quantity, Column 2 and Row 2:

Normalisation, Column 3: scale factor to be applied to scaling because of results of full model,

Column 4 to 13: Exponent of power law scaling for quantity at head of the column. $\xi_{\mathrm{ei}}$ is ratio of power carried by electrons to that carried by ions ( 1 for all cases here). $[\mathrm{n}]$ is $10^{20} \mathrm{~m}^{-3},[\Gamma]$ is $\mathrm{Pa}-\mathrm{m}^{3} / \mathrm{s},[\mathrm{P}]$ is $\mathrm{MW}$. 


\section{Figure Captions}

Fig. 1 Normalised divertor pressure and separatrix DT density (without size scaling) versus radius of outer strike point $\mathrm{R}_{\text {outer }}$ at incipient detachment of the inner divertor, $\mu=1$, for devices indicated and for SOL powers from small (circle), medium (lozenge) to high (square). Powers are: AUG: 2, 4, 8 MW, JET: 8, 16, 24 MW, ITER: 86, 100, 130 MW, DEMO: 200, 400, 500 MW. The exponent of the fit on the log-log scale is indicated on the figure as " $m=\ldots$ (e.g. $\mathrm{m}=1.21$ for left pane of Fig. 1.)

Fig. 2 Normalised DT and He neutral influx at separatrix versus $\mathrm{R}_{\text {outer }}$ at $\mu=1$. See caption Fig. 1.

Fig. 3 Normalised He density separatrix and peak power per unit area at divertor plate versus $\mathrm{R}_{\text {outer }}$ at $\mu=1$. See caption Fig. 1 .

Fig. 4 Normalised helium density and helium neutral influx at separatrix, including $\mathrm{R}$ dependence, versus normalised divertor neutral pressure $\mu$, for one ITER and one DEMO case, for both linear neutral model (unmarked) and complete neutral model (see text, marked "NF").

Fig. 5 Scrape-off layer power and volume-averaged electron density for DEMO versus Xe seed concentration (\%). Peak power of $10(7) \mathrm{MW} / \mathrm{m}^{2}$ indicated by square(lozenge).

Fig. 6 a) Normalised peak power per unit area on the divertor plates including $\mathrm{R}$ dependence, versus normalised divertor neutral pressure $\mu$, for JET, ITER, and Demo, b) Peak power per unit area on the divertor plates vs. seed Xe concentration. Peak power of $10(7) \mathrm{MW} / \mathrm{m}^{2}$ indicated by square(lozenge). 


\section{Figures}

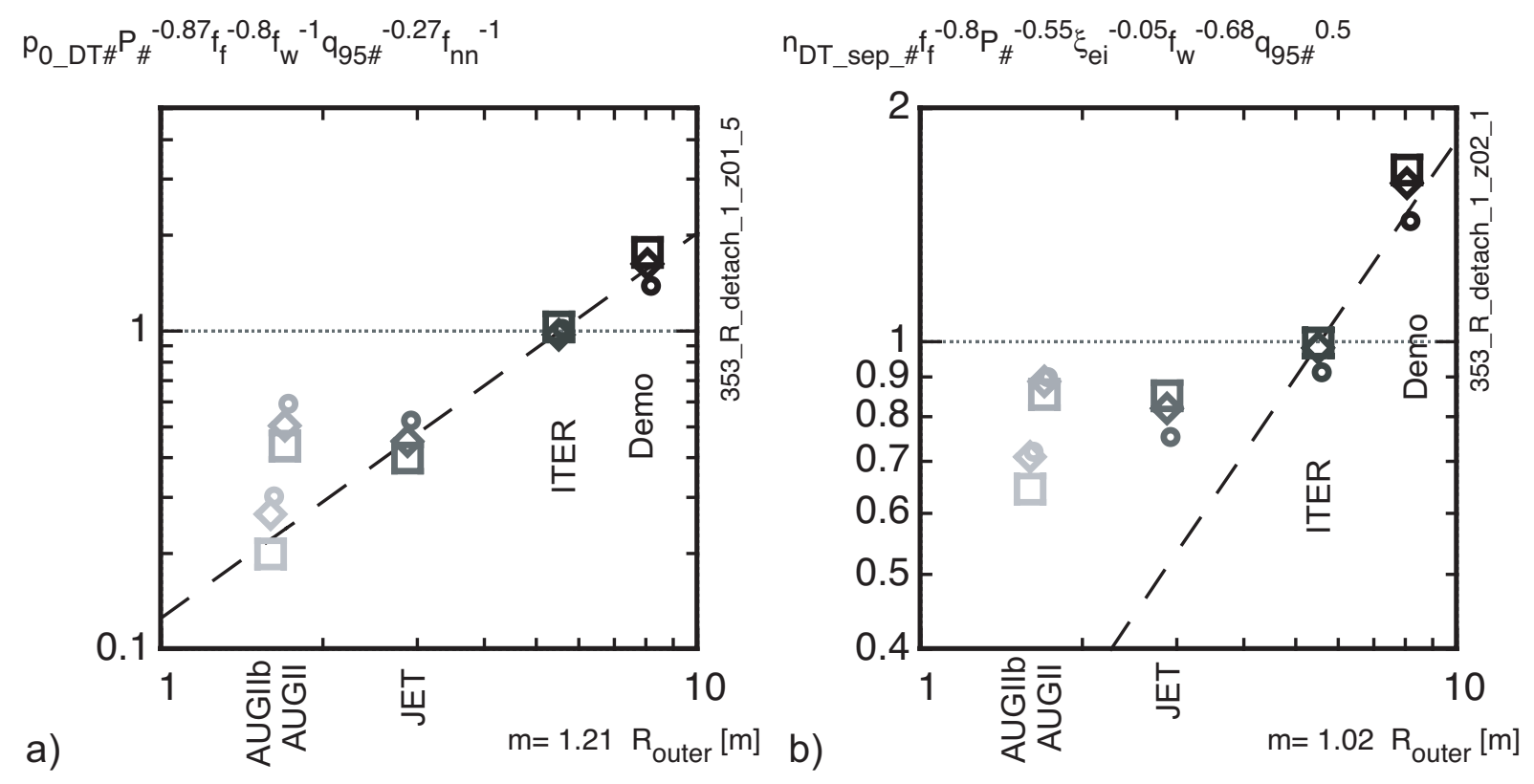

Fig. 1 


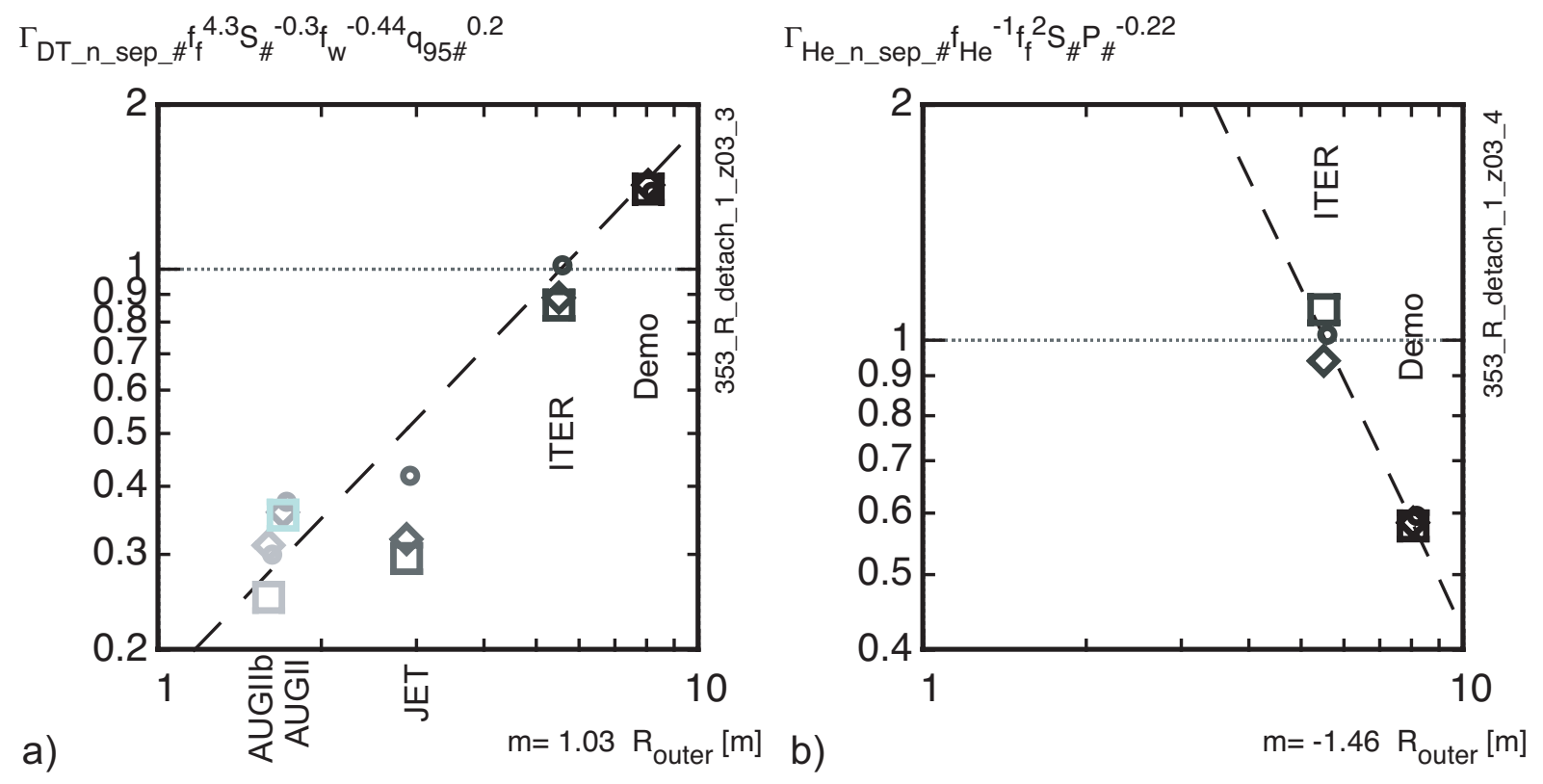

Fig.2 


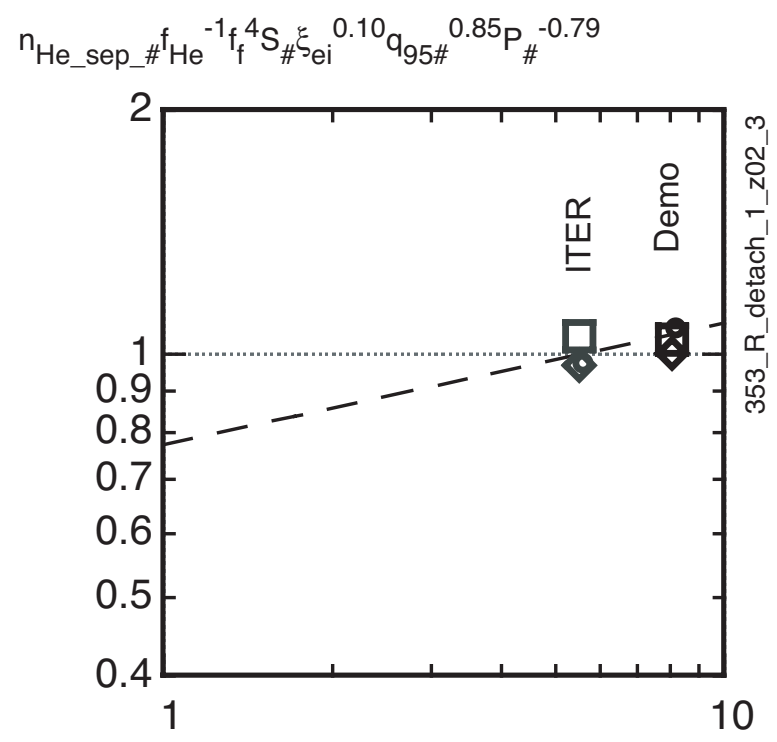

a)

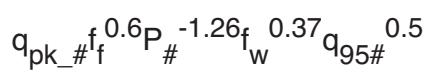

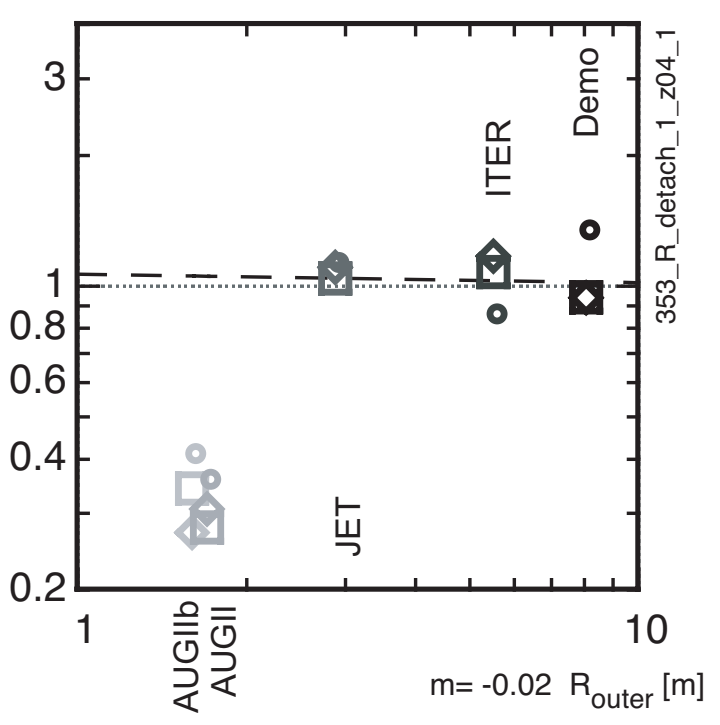

Fig. 3 


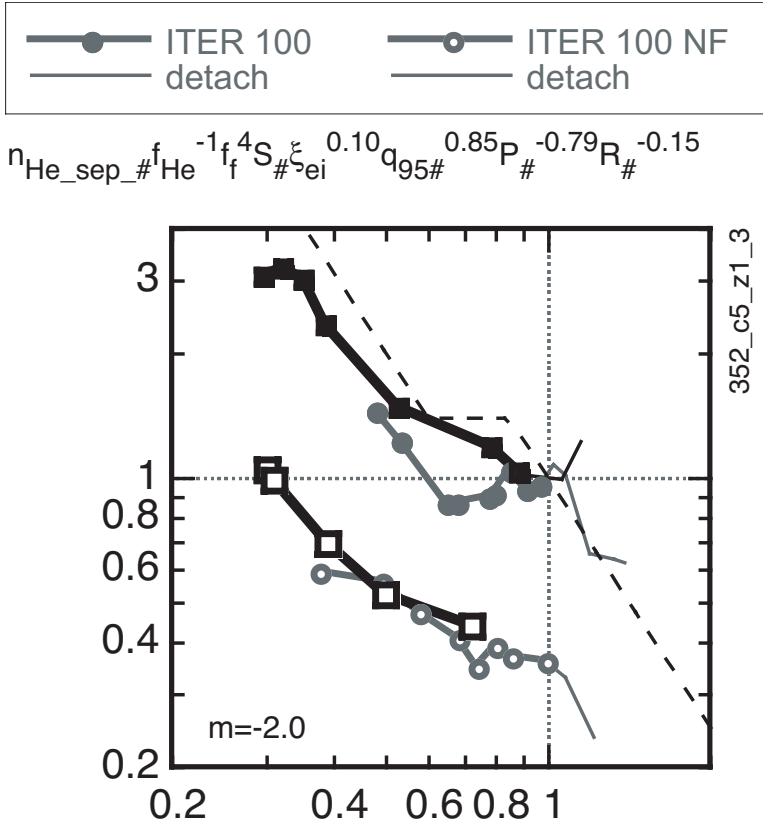

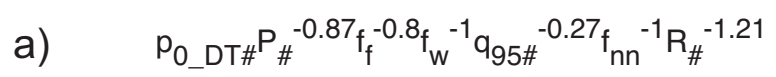

Demo $200 \quad-\mathbf{D}$ - Demo $200 \mathrm{NF}$
detach

$\Gamma_{\text {He_n_sep_\# }} \mathrm{f}^{-1} \mathrm{f}_{\mathrm{f}}{ }^{2} \mathrm{~S}_{\#} \mathrm{P}_{\#}{ }^{-0.22} \mathrm{R}_{\#}{ }^{1.46}$

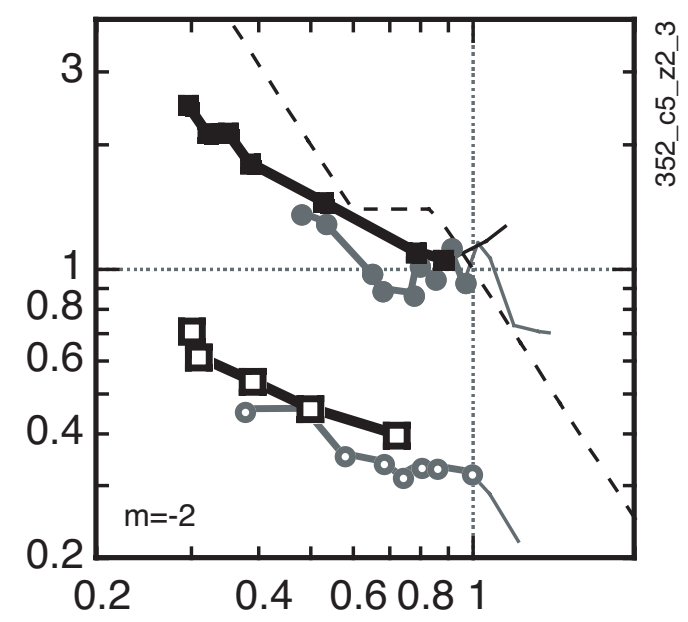

b) $\quad \mathrm{p}_{0 \_D T \#} P^{-0.87_{\mathrm{f}}}-0.8 \mathrm{f}_{\mathrm{f}}{ }^{-1} \mathrm{q}_{95 \#}{ }^{-0.2 \mathrm{f}_{\mathrm{nn}}}{ }^{-1} \mathrm{R}_{\#}{ }^{-1.21}$

Fig. 4 

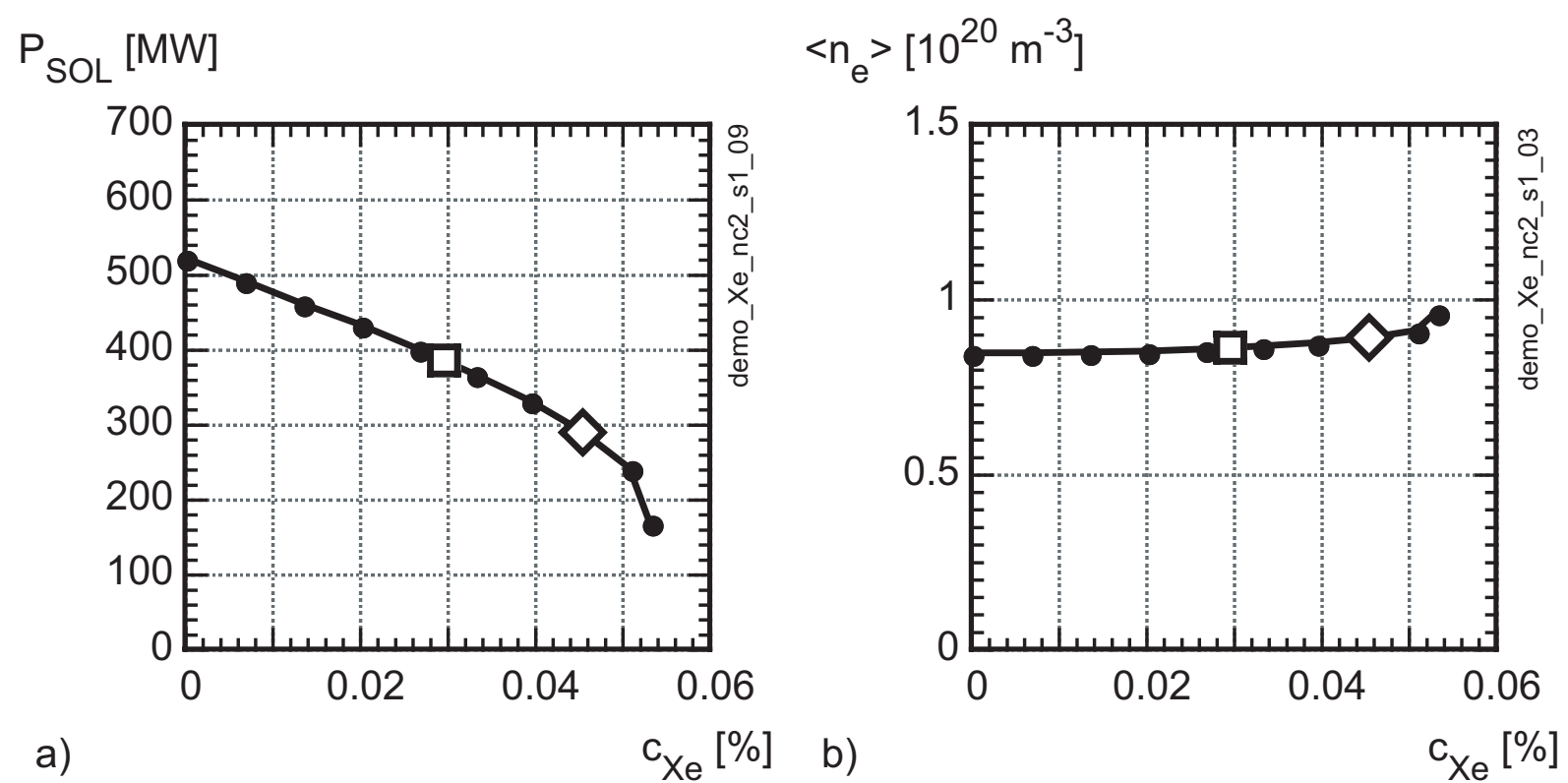

Fig. 5 


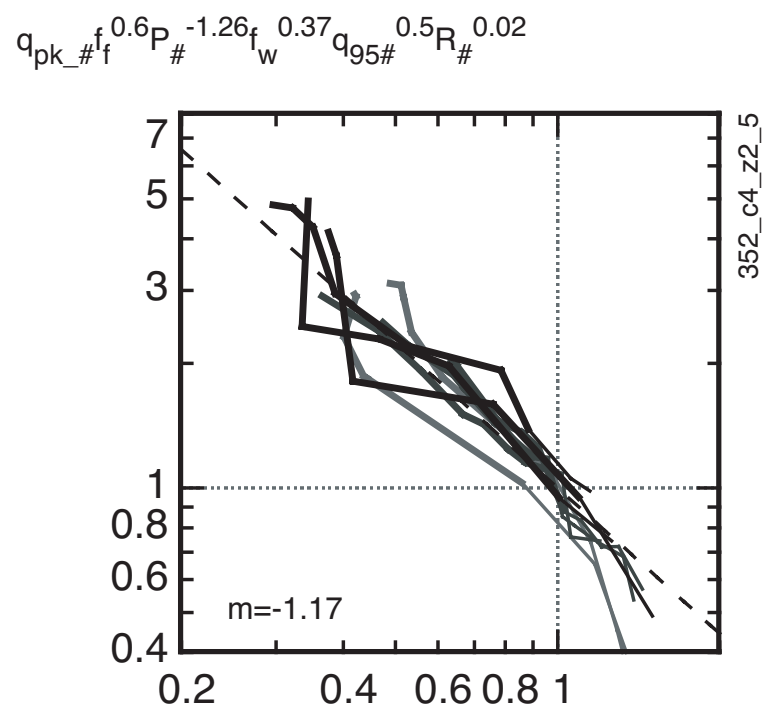

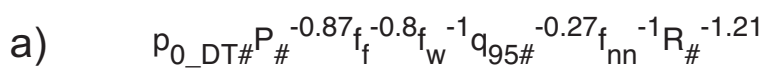

Fig. 6

$$
\mathrm{q}_{\mathrm{pk}}\left[\mathrm{MW} / \mathrm{m}^{2}\right]
$$

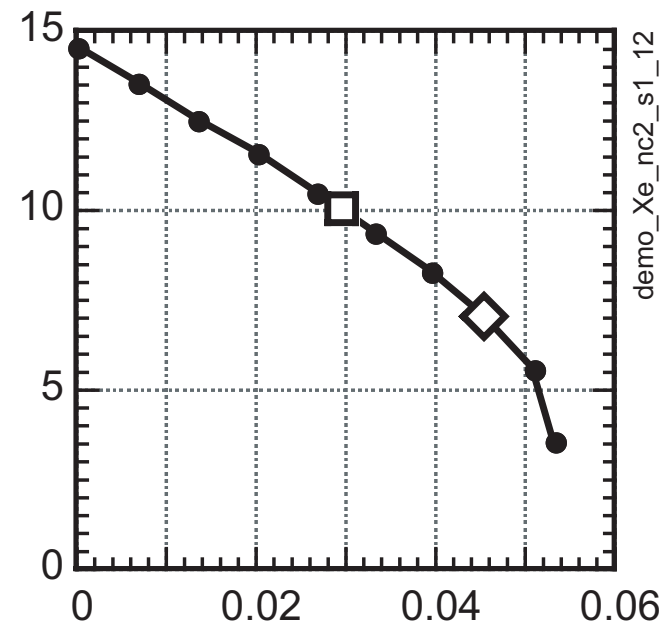

b) ${ }^{\mathrm{c}} \mathrm{e}^{[\%]}$ 